\title{
Summer sleep quality and change of bedroom thermal environment - from the beginning to the end of sleep
}

\author{
Noriko Umemiya ${ }^{1, *}$, Kurumi Yamagata $^{1}$ and Tomohiro Kobayashi ${ }^{1}$ \\ ${ }^{1}$ Osaka City University, Graduate School of Engineering, 558-8585 3-3-138 Sugimoto, Sumiyoshi, Osaka City, Japan
}

\begin{abstract}
Relations between bedroom thermal environment changes and subjective sleep quality and thermal comfort were surveyed for 63 apartment occupants during midsummer in Osaka. Changes of Wet Bulb Globe Temperature (WBGT) of 123 air-conditioned and 41 naturally ventilated nights were compared. 1) For air-conditioned bedrooms, sleep quality was better for smaller changes of WBGT from the beginning of sleep and for faster changes of WBGT to constant from the beginning of sleep. 2) For naturally ventilated bedrooms, a) WBGT increased from the beginning of sleep and decreased toward the end of sleep for higher sleep quality nights. However, WBGT decreased from the beginning of sleep and increased toward the end of sleep for lower sleep quality nights. b) The WBGT change was smaller for thermally comfortable nights.
\end{abstract}

\section{Purpose}

Preserving sleep quality is an important issue during hot and humid summers, especially in urban crowded areas affected by heat island phenomena. Air conditioning is not preferred for several reasons. Appropriate modes of air conditioner use are required.

Tachibana et al. (2017) compared the use of air conditioning all night, the use of natural ventilation all night, and the use of air conditioning for part of the night for apartment occupants in Osaka during summer. Results show that 1) thermal sensations were not different among the three patterns, and 2) subjective sleep quality of natural ventilation all night was better than for the use of air conditioning all night. It compared only averaged thermal environments during sleep [1]. The present study analysed effects of thermal environment changes during sleep on thermal sensation and subjective sleep quality.

\section{Methods}

\subsection{Measurement procedure}

Surveys of 63 occupants in apartment buildings were conducted for seven successive nights during midsummer in 2017. They measured bedside temperatures at $10 \mathrm{~min}$ intervals. Relative humidity was recorded before and after sleep. Every morning, the occupants filled in questionnaire sheets with information about the prior night, reporting sleep quality in bedrooms on 15 items using four-point-scales, thermal sensation on a seven- point-scale, thermal comfort on a four-point-scale, and thermal acceptability on a three-point-scale. They also maintained a diary recording phenomena at $30 \mathrm{~min}$ intervals: their absence or presence in the bedroom, personal air conditioner use, electric fan use, window opening in the bedroom, and sleeping or awakening. Subjective sleep quality was evaluated using the OSA sleep quality inventory.

\subsection{OSA sleep quality inventory}

Oguri, Shirakawa and Azumi developed the eponymous OSA sleep quality inventory in 1985 based on data of nearly seven hundred subjects. It evaluates sleep subjectively at the time of awakening based on responses to 16 questions given using four-point-scales: very good, somewhat good, somewhat bad, or very bad [2-3].

The 16 responses were classified into five factors: Factor I - Drowsiness when waking such as 'I have the power of concentration.', 'I feel a sense of liberation.', and 'I feel clear-headed.'; Factor II - Falling asleep and maintaining sleep such as 'I was able to sleep soundly.', 'I dozed off until I finally fell asleep.', 'I got to sleep easily.', 'I often woke up from sleep.', and 'The sleep was shallow.'; Factor III - Dreaming, such as 'I had many nightmares.' and 'I had many dreams.'; Factor IV - Fatigue recovery such as 'Fatigue persists after waking up.', 'I feel languorous.', and 'I feel unwell.'; and Factor V - Sleeping duration such as 'I have generally good appetite.' and 'The sleep duration was long.' The OSA score is an average of five factors of a T-score.

Higher OSA scores reflect higher sleep quality.

\footnotetext{
* Corresponding author: umemiyanor@osaka-cu.ac.jp
} 


\subsection{Wet Bulb Globe Temperature}

The Wet Bulb Globe Temperature (WBGT) was estimated from measured air temperature and relative humidity. Average relative humidity measured before and after sleep was used.

The Wet Bulb Globe Temperature is an index representing human heat stress in hot environment standardized by ISO7243 [4]. Reference WBGT values for sports activity are shown by the Japan Sports Association: WBGT 21_25 $5^{\circ} \mathrm{C}$ for 'attention', $25 \_28^{\circ} \mathrm{C}$ for 'warning', $28 \_31^{\circ} \mathrm{C}$ for 'strong warning' and $31^{\circ} \mathrm{C}$ for 'discontinuation' [5]. The Japan Institute of Biometeorology recommends other reference values indoors in daily life in moderate radiation and air movement that is estimated by room air temperature and humidity [6]. Japan's ministry of the environment estimates and releases the WBGT value every day in hot seasons to call attention to heatstroke [7].

WBGT is more suitable to estimate bedroom thermal environment than air temperature because it is a synthetic thermal index incorporating radiation, humidity, and air velocity.

\subsection{Distribution of Measurements}

Fig. 1 shows the daily mean, maximum and minimum outdoor temperatures measured at Osaka meteorological observatory [8]. The survey was conducted in midsummer because there was no trend in daily outdoor temperatures. Furthermore, little trend was apparent in the daily numbers of measurements.

\section{Results}

\subsection{Occupants}

Occupants were $27-88$ years old; $62.1 \%$ were women. Fig. 2 shows a frequency distribution of OSA scores. Higher scores over 50 were dominant over lower scores. The mean OSA score was 52.3.

\subsection{Thermal control use patterns}

Fig. 3 shows a frequency distribution of thermal control use patterns. In the figure, ' $O$ ' shows 'full time use', ' $\Delta$ ' denotes 'part time use', and ' $\mathrm{X}$ ' represents 'no use'. The three successive marks respectively show the use of an air conditioner, natural ventilation, and an electric fan. The frequent use patterns were 123 nights of air conditioner use all night without natural ventilation or an electric fan (OXX), 57 nights of partial air-conditioner use without natural ventilation or an electric fan $(\Delta X X)$, 43 nights of electric fan use with natural ventilation (XOO), and 41 nights of natural ventilation use on all

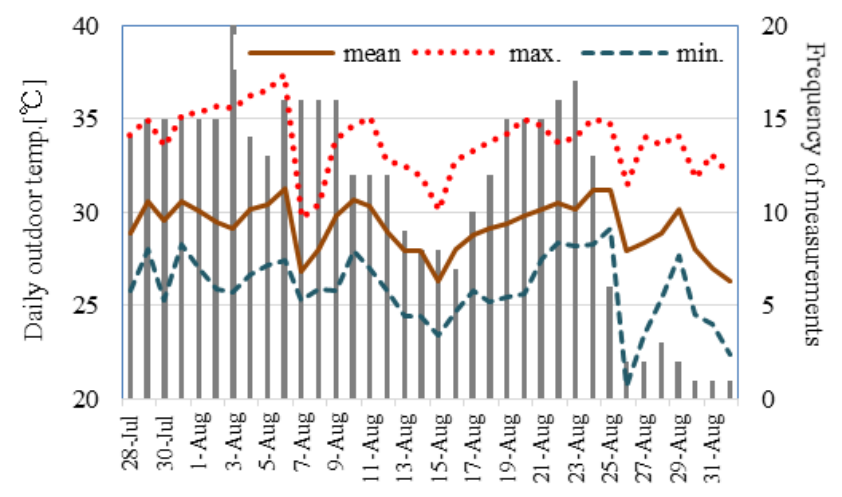

Fig.1. Daily outdoor temperature and number of measured nights

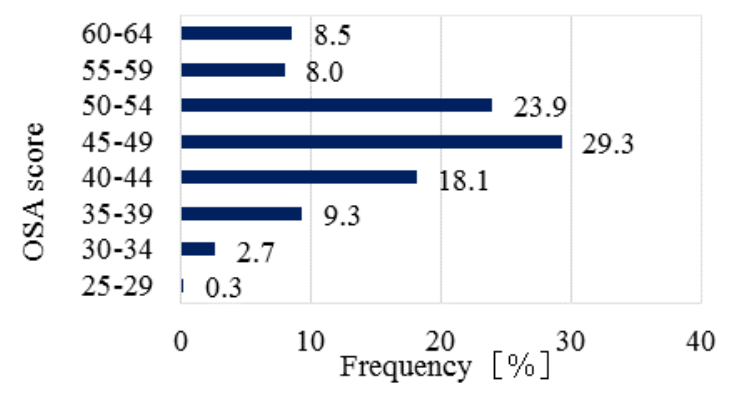

Fig.2. Distribution of OSA score

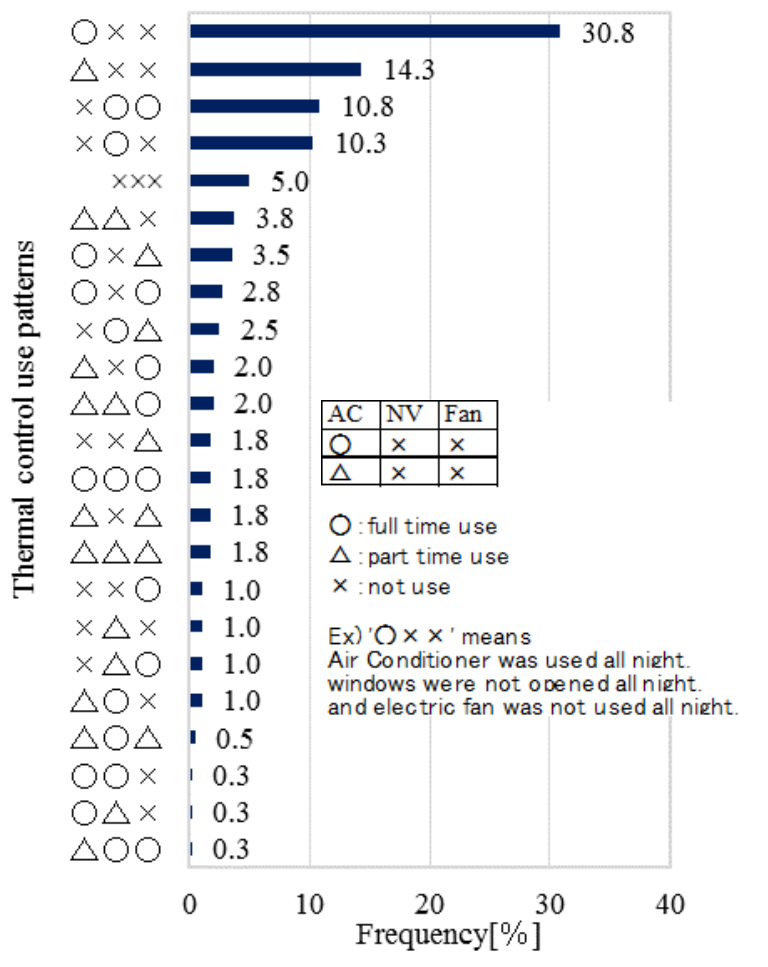

Fig.3. Distribution of thermal control patterns 

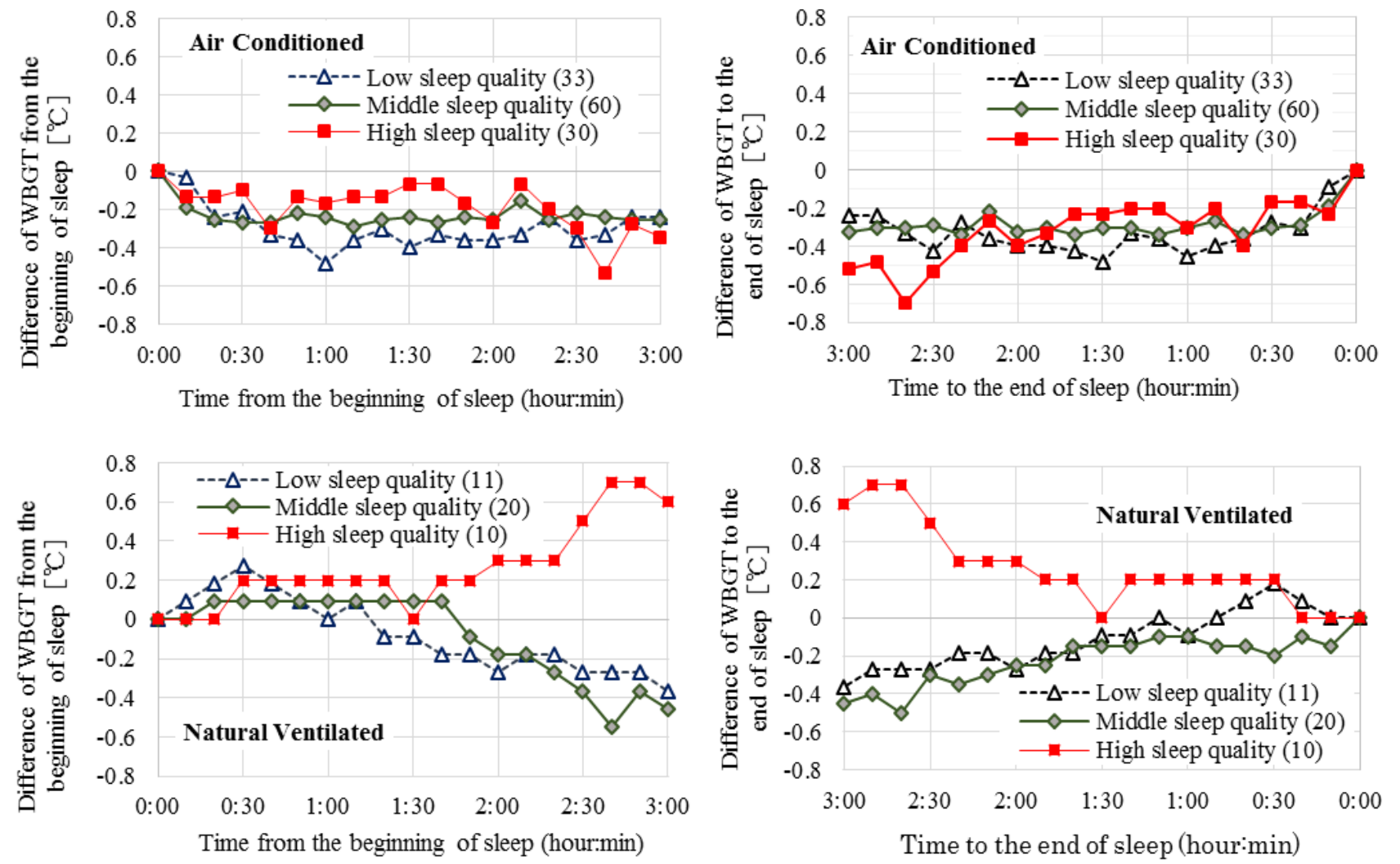

Fig.4. WBGT change from the beginning of sleep for different sleep quality levels

Fig.6. WBGT change to the end of sleep for different sleep quality levels
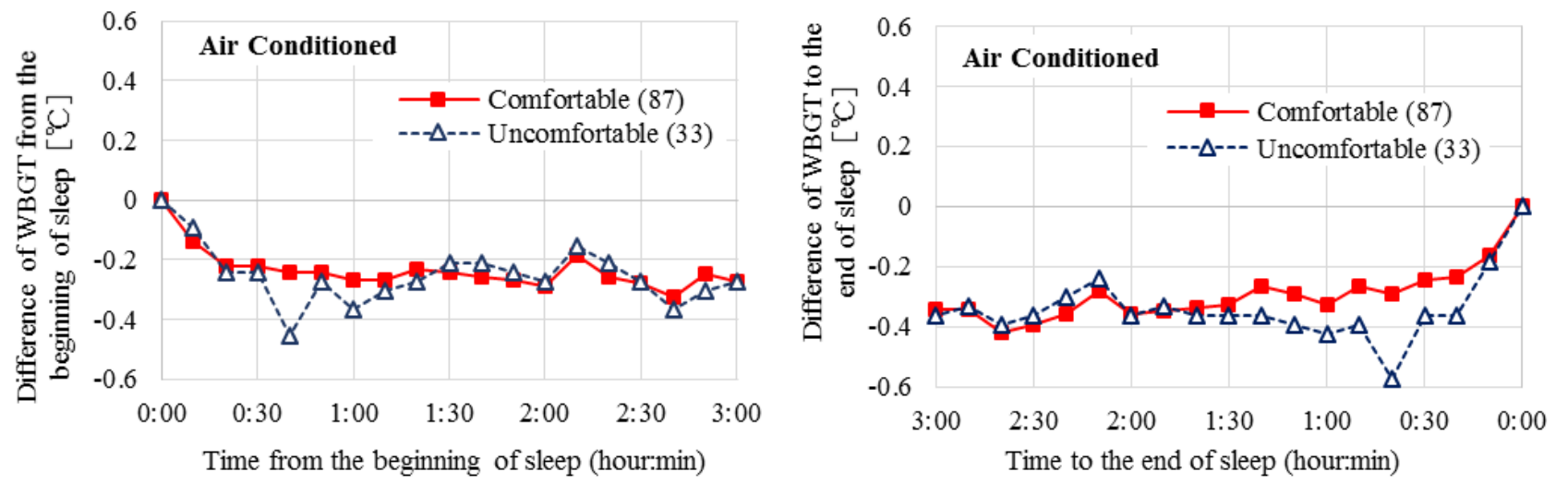

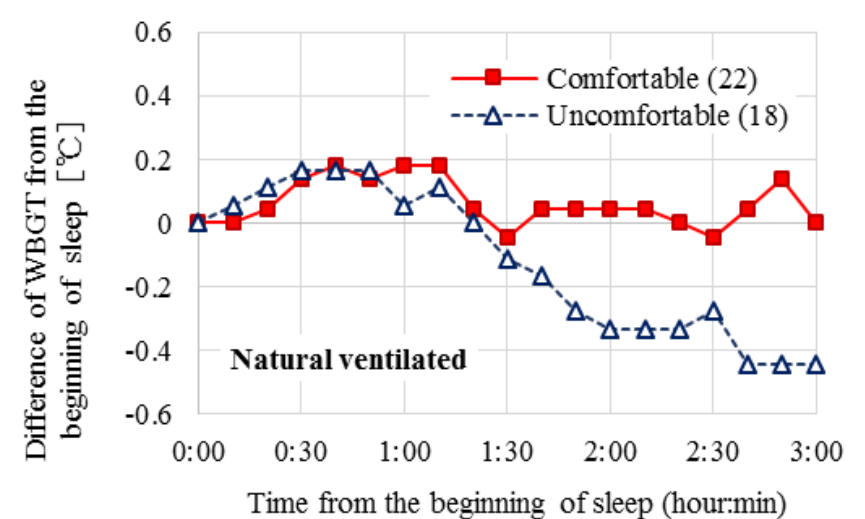

Fig.5. WBGT change from the beginning of sleep for thermally Comfortable and Uncomfortable nights

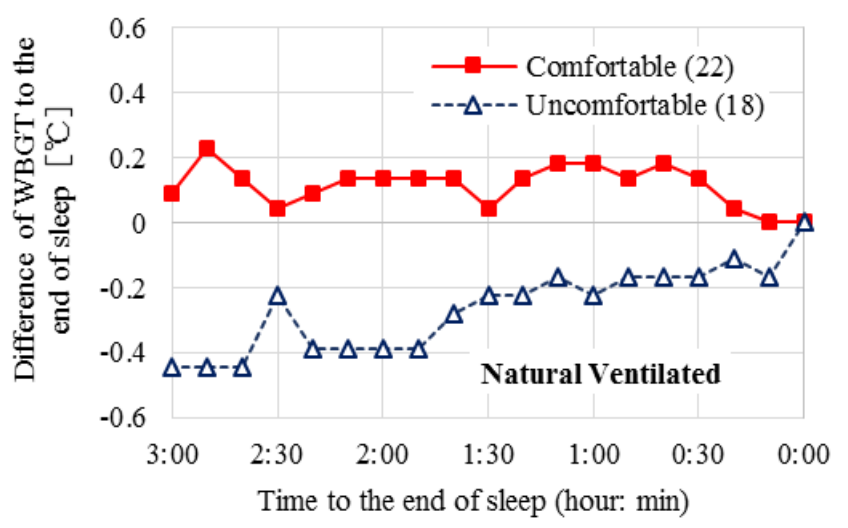

Fig.7. WBGT change to the end of sleep for thermally Comfortable and Uncomfortable nights 
nights (XOX). All relative frequencies were over $10 \%$, except for that of the fifth pattern, which was $5.0 \%$. The other four patterns can be regarded as frequent patterns. We compare 123 ' $\mathrm{XOO}$ ' and 41 'XOX' in this study to clarify differences between air conditioning and natural ventilation.

\section{Change of WBGT from the beginning of sleep}

Nights were classified according to the OSA score: high OSA nights had an OSA score higher than $75 \%$ of the OSA value; middle OSA nights had an OSA score of $25 \%-75 \%$ of the OSA value; and low OSA nights had an OSA score lower than $25 \%$ of the OSA value. They were classified for the respective control patterns. Nights were also classified according to thermal comfort.

We examined changes from the beginning of sleep. Specifically, we assessed phenomena at three hours from the beginning of sleep and three hours toward the end of sleep because almost all occupants slept for more than three hours.

Thermal sensation and thermal acceptability were not related to the WBGT change from the beginning of sleep both for air conditioned case and for natural ventilated case. But sleep quality score and thermal comfort were related to the WBGT change from the beginning of sleep in some cases.

\subsection{Change of WBGT and sleep quality}

Fig. 4 shows the change of WBGT from the beginning of sleep for Low OSA score (under 25 percentile value) nights, Middle OSA score (25-75 percentile value) nights, and High OSA score (over 75 percentile value) nights. WBGT was averaged for each $10 \mathrm{~min}$ for nights classified into the respective OSA score groups. Values in parenthesis in figures show the number of nights in the groups.

\subsubsection{Air conditioned case}

From the beginning of sleep, WBGT decreased and became constant. The WBGT differences from the beginning were $0.1^{\circ} \mathrm{C}, 0.2^{\circ} \mathrm{C}$ and $0.4^{\circ} \mathrm{C}$, respectively, for High, Middle, and Low OSA score nights. It became constant $15 \mathrm{~min}, 30 \mathrm{~min}$, and $60 \mathrm{~min}$, respectively, after the beginning for High, Middle, and Low nights. Change was smaller for higher OSA score nights and became constant faster for higher OSA score nights. Sleep quality was better when the decrease of WBGT was smaller and faster to be constant for air-conditioned nights.

\subsubsection{Natural ventilated case}

WBGT increased after the beginning of sleep, and subsequently decreased. It began to decrease after 100 min and $30 \mathrm{~min}$, respectively, for Middle and Low OSA score nights. It became lower than the beginning at 110 min and $80 \mathrm{~min}$ after the beginning. However, it continued increasing for $3 \mathrm{hr}$ from the beginning of sleep for High OSA score nights. Sleep quality was low when WBGT decreased. It was better when WBGT increased from the beginning for natural ventilated nights.

\subsection{Change of WBGT and thermal comfort}

Fig. 5 shows the change of WBGT from the beginning of sleep for thermally Comfortable nights ('comfortable') and Uncomfortable nights ('slightly uncomfortable', 'uncomfortable' and 'very uncomfortable') when air conditioned and natural ventilated. WBGT was averaged for each $10 \mathrm{~min}$. Values in parenthesis in figures show the number of nights in the groups.

\subsubsection{Air conditioned case}

WBGT decreased $0.2^{\circ} \mathrm{C}$ during $30 \mathrm{~min}$ from the beginning of sleep and then became constant for both thermally Comfortable and Uncomfortable nights. Thermal comfort was unrelated to the change of WBGT from the beginning of sleep when air conditioned.

\subsubsection{Natural ventilated case}

For thermally Comfortable and Uncomfortable nights, WBGT changed similarly after $90 \mathrm{~min}$ from the beginning. It became $0.2^{\circ} \mathrm{C}$ higher than the beginning after $40 \mathrm{~min}$ from the sleep beginning. Then WBGT became $0.2^{\circ} \mathrm{C}$ lower than the beginning after $70-90 \mathrm{~min}$ from the beginning. After that, WBGT changed differently between thermally Comfortable and Uncomfortable nights. It became the same to the beginning for Comfortable nights. However, it became $0.2-0.4^{\circ} \mathrm{C}$ lower than the beginning for Uncomfortable nights. The width of the WBGT change was larger for thermally Uncomfortable nights. The WBGT change was found not to affect thermal comfort until $90 \mathrm{~min}$ after the beginning of sleep, but it continued descending after 90 min for thermally Uncomfortable nights.

\section{Change of WBGT toward the end of sleep}

We examined changes toward the end of sleep. Specifically, we assessed phenomena at three hours toward the end of sleep because almost all occupants slept for that duration.

\subsection{Change of WBGT and sleep quality}

Fig. 6 shows the change of WBGT toward the end of sleep for Low OSA score nights (under 25\%), Middle OSA score nights (25-75\%), and High OSA score nights (over 75\%) nights when air conditioned and natural ventilated. WBGT was averaged for each $10 \mathrm{~min}$.

Thermal sensations and thermal acceptability were not related to WBGT changes toward the end of sleep. But 
sleep quality score and thermal comfort were related to the WBGT changes towards the end of sleep in some cases.

\subsubsection{Air conditioned case}

Results show that WBGT at $3 \mathrm{hr}$ before the end of sleep was lower than that of the end of sleep, irrespective of the OSA score. It began to increase after $30 \mathrm{~min}$ before the end of sleep. The difference was smaller for higher OSA at $3 \mathrm{hr}$ before the end. However, the order reversed at $100 \mathrm{~min}$ before the end and was maintained. Sleep quality was high for smaller WBGT difference until 90 min before the end, but the relation reversed thereafter.

\subsubsection{Natural ventilated case}

The WBGT of High OSA score nights was $0.6^{\circ} \mathrm{C}$ higher than that at $3 \mathrm{hr}$ before the end of sleep. It reached $0.7^{\circ} \mathrm{C}$ and then decreased to $0.2^{\circ} \mathrm{C} 110 \mathrm{~min}$ before the end and became equal to the end value at $20 \mathrm{~min}$ before the end. WBGT of Middle OSA score nights remained lower than the end WBGT, although it increased gradually from lower than $0.5^{\circ} \mathrm{C}$ at the end. The WBGT of Low OSA score nights was $0.4^{\circ} \mathrm{C}$ lower. It became $0.2^{\circ} \mathrm{C}$ higher than the end and subsequently decreased. The width of WBGT change was $0.7^{\circ} \mathrm{C}, 0.5^{\circ} \mathrm{C}$, and $0.6^{\circ} \mathrm{C}$, respectively, for High, Middle, and Low OSA score nights.

\subsection{Change of WBGT and thermal comfort}

Fig. 7 shows the change of WBGT toward the end of sleep for thermally Comfortable nights ('comfortable') and Uncomfortable nights ('slightly uncomfortable', 'uncomfortable' and 'very uncomfortable') when air conditioned and natural ventilated.

\subsubsection{Air conditioned case}

There was little difference in the change of WBGT between thermally Comfortable and Uncomfortable nights until 90 min before the end of sleep. Results show that WBGT was $0.3^{\circ} \mathrm{C}$ lower than at the end and constant until $90 \mathrm{~min}$ before the end. Then it began to increase gradually until $30 \mathrm{~min}$ before the end. It was $0.4^{\circ} \mathrm{C}$ lower than the end and became $0.6^{\circ} \mathrm{C}$ lower than the end at $40 \mathrm{~min}$ before the end and subsequently increased to the end. The WBGT change width was larger for thermally Uncomfortable nights when using air conditioning.

\subsubsection{Natural ventilated case}

WBGT was $0-0.2^{\circ} \mathrm{C}$ higher than at the end of sleep for thermally Comfortable nights and $0.4-0^{\circ} \mathrm{C}$ lower $3 \mathrm{hr}$ before the end than at the end of sleep for thermally Uncomfortable nights. The WBGT change width was larger for Uncomfortable nights, similarly to air conditioned cases.

\section{Conclusion}

For 63 occupants of apartments in midsummer in Osaka bedrooms, the authors surveyed the bedroom thermal environment, thermal sensation, thermal comfort, thermal acceptability, and subjective sleep quality. The relation between WBGT change in bedrooms from the beginning of sleep and toward the end of sleep and thermal sensation, thermal comfort, thermal acceptability and sleep quality were compared using data from 123 air conditioned and 41 naturally ventilated cases.

Results show the following. 1) If bedrooms were air conditioned, sleep quality was better for smaller changes of WBGT from the beginning of sleep and was better for faster changes of WBGT to a constant from the beginning of sleep. 2) For naturally ventilated bedrooms, a) WBGT increased from the beginning of sleep and decreased toward the end of sleep for higher sleep quality nights. However, WBGT decreased from the beginning of sleep and increased toward the end of sleep for lower sleep quality nights. b) The WBGT change was smaller for thermally comfortable nights. 3) Thermal sensations and thermal acceptability during sleep were not related to WBGT changes in bedrooms.

Special thanks to Jun-Ichiro Arai, DAIKIN INDUSTRIES, Ltd..

\section{References}

1. Y. Tachibana, N. Umemiya, T. Kobayashi, H. Bessho, Y. Nakayama, Relation between thermal control use and sleep quality during summer Survey of apartments in Osaka, Proceedings of Annual Meeting of Kinki Chapter of Society of Heating, Air Conditioning and Sanitary Engineers of Japan, 46, A-56 (2017) (in Japanese)

2. M. Oguri, S. Shirakawa, K. Azumi, Development of OSA sleep quality inventory - Statistical construction and standardization of rating scale for evaluation of subjective sleep sensation. Clinical Psychiatry, 27(7), 791-798 (1985)

3. Y. Yamamoto, H. Tanaka, M. Takahashi, K. Yamasaki, K. Azumi, S. Shirakawa, Standardizationof revised version of OSA sleep inventory for middle age and aged, Brain Science and Mental Disorders, 10, 401-409 (1999)

4. ISO, ISO7243: Hot environments - Estimation of the heat stress on working man, based on the WBGT-index (wet bulb globe temperature) (1989)

5. Japan Sports Association, (2004). Guideline for heatstroke prevention in sports activities.

6. Guideline for Prevention of Heat Stroke in Daily Life, Japanese Society of Biometeorology (2013)

7. Japan Ministry of Environment, Heat illness prevention information, http://www.wbgt.env.go.jp/

8. Japan Meteorological Agency, http:// www.jma.go. jp /jma/menu/menureport.html 